\title{
SEEG Trajectory Planning: Combining Stability, Structure and Scale in Vessel Extraction
}

\author{
Maria A. Zuluaga ${ }^{1}$, Roman Rodionov ${ }^{2,3}$, Mark Nowell ${ }^{2,3}$, Sufyan Achhala ${ }^{2}$, \\ Gergely Zombori ${ }^{1}$, M. Jorge Cardoso ${ }^{1}$, Anna Miserocchi ${ }^{2,3}$, \\ Andrew W. McEvoy ${ }^{2.3}$, John S. Duncan ${ }^{2,3}$, and Sébastien Ourselin ${ }^{1}$ \\ 1 Translational Imaging Group, CMIC, University College London, London UK \\ 2 Dept. of Clinical and Experimental Epilepsy, UCL IoN, London, UK \\ ${ }^{3}$ National Hospital for Neurology and Neurosurgery (NHNN), London, UK
}

\begin{abstract}
StereoEEG implantation is performed in patients with epilepsy to determine the site of the seizure onset zone. Intracranial haemorrhage is the most common complication associated to implantation carrying a risk that ranges from 0.6 to $2.7 \%$, with significant associated morbidity [2]. SEEG planning is done pre-operatively to identify avascular trajectories for the electrodes. In current practice neurosurgeons have no assistance in the planning of the electrode trajectories. There is great interest in developing computer assisted planning systems that can optimize the safety profile of electrode trajectories, maximizing the distance to critical brain structures. In this work, we address the problem of blood vessel extraction for SEEG trajectory planning. The proposed method exploits the availability of multi-modal images within a trajectory planning system to formulate a vessel extraction framework that combines the scale and the neighbouring structure of an object. We validated the proposed method in twelve multi-modal patient image sets. The mean Dice similarity coefficient (DSC) was $0.88 \pm 0.03$, representing a statistically significantly improvement when compared to the semi-automated single rater, single modality segmentation protocol used in current practice (DSC $=0.78 \pm 0.02$ ).
\end{abstract}

\section{Introduction}

Stereo-electroencephalography (SEEG) is the recording of the brain electrical activity by depth electrodes implanted into the brain parenchyma. SEEG is indicated in patients with medically refractory epilepsy who are candidates for epilepsy surgery. The purpose of SEEG is to precisely identify the area of the brain where seizures start, known as the seizure onset zone (SOZ) [1]. The major complication with SEEG implantation is intracranial haemorrhage [2]. Therefore, preoperative SEEG planning is a necessary prerequisite to implantation. The aim of planning is to identify electrode trajectories that achieve adequate cortical coverage and pass through safe avascular planes.

In recent years there has been great interest in the development of computer assisted planning systems for optimizing intracranial depth electrode in- 
sertion [3-6]. These methods rely on effective extraction of critical brain landmarks with high accuracy and robustness. In this work, we specifically address the extraction of the intracranial vasculature within an SEEG planning system.

Despite years of research [7], methods of vessel extraction still tend to suffer from discontinuities caused by low intensity from partial volume or noise. A common solution is the use of a vesselness filter $[8,9]$ that enhances voxels within tubular structures. These filters have been very successful through the inclusion of scale within their formulation, but lack information about neighbouring voxel structure. Also, despite increased access to multi-modal images, very few methods have exploited the information redundancy to improve vessel extraction. In [10], Passat et al. combined multiple MR sequences to segment the superior sagittal sinus. However, vessel extraction was only performed on a single image, with a second modality used to provide a priori anatomical information of the brain. More recently, Hu et al. [11] proposed the first true multi-modal approach to vessel extraction by using features extracted from optical coherence tomography and fundus photography with a k-NN classifier to segment $2 \mathrm{D}$ retinal vessel images.

In this paper, we present a novel method that integrates the concepts of scale, neighbourhood structure and feature stability with the aim of improving the robustness and accuracy of vessel extraction within a computer assisted SEEG planning system [12]. The method accounts for both the scale and vicinity of a voxel by formulating the problem within a multi-scale tensor voting framework. Feature stability is achieved by introducing a similarity measure that evaluates the multi-modal consistency in the vesselness responses. The proposed measurement allows the combination of the multiple responses into a unique image that is then used within the planning system to visualize critical vessels.

\section{Method}

The tensor voting framework [13] is a robust technique for extracting structures from a cloud of points. It is based on the principle that a set of unconnected tokens (i.e. points) can exchange information within a neighbourhood that allows one to infer the geometric structure in which a token lies. In 3D, it enables an estimation of saliency measurements of the likelihood that a token lies on a surface, a curve, a junction or is just noise.

Tensor voting consists of three stages: token initialisation, tensor voting and voting result analysis. In order to give our method feature stability and scale variance, we add a data fusion step, and we embed all this into a multi-scale framework. A diagram illustrating the proposed method is shown in Figure 1.

Token Initialisation Under the tensor voting formalism, information contained by a token $\mathbf{p}$ is encoded in a 3D second order, symmetric, non-negative definite tensor $\mathbf{T}$, which is equivalent to a $3 \times 3$ matrix and a $3 \mathrm{D}$ ellipsoid. According to the spectrum theorem, $\mathbf{T}$ can be expressed as the linear combination 


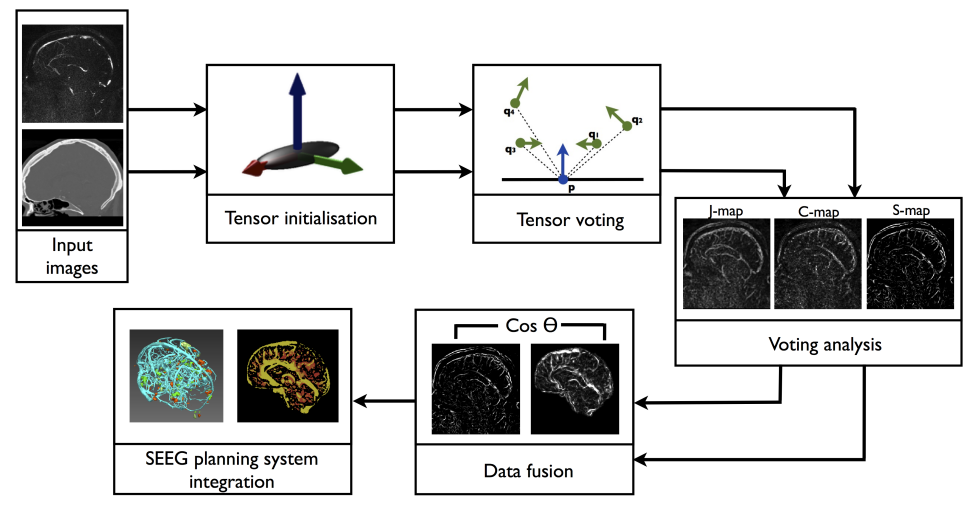

Fig. 1. Vessel extraction diagram at a single scale. Two images are converted into tokens through analysis of the Hessian matrix. After voting, the resulting saliency maps are combined using the cosine between the vectors defining orientation. As the approach is performed within a multi-scale framework, the maximum response obtained within a range of scales is kept and visualised in the planning system.

of three tensors:

$$
\mathbf{T}=\left(\lambda_{1}-\lambda_{2}\right)\left(\mathbf{e}_{\mathbf{1}} \mathbf{e}_{\mathbf{1}}^{T}\right)+\left(\lambda_{2}-\lambda_{3}\right) \sum_{i=1}^{2} \mathbf{e}_{\mathbf{i}} \mathbf{e}_{\mathbf{i}}^{T}+\lambda_{3} \sum_{i=1}^{3} \mathbf{e}_{\mathbf{i}} \mathbf{e}_{\mathbf{i}}^{T}
$$

where $\lambda_{i}$ are the tensor's eigenvalues in decreasing order and $\mathbf{e}_{\mathbf{i}}$ the corresponding eigenvectors.

The tensors in Eq. 1 provide different structural information. The first term is a stick tensor $S=\left(\lambda_{1}-\lambda_{2}\right)\left(\mathbf{e}_{1} \mathbf{e}_{1}^{T}\right)$, encoding eccentricity with orientation $\mathbf{e}_{\mathbf{1}}$. The second term is a plate tensor $P=\left(\lambda_{2}-\lambda_{3}\right) \sum_{i=1}^{2} \mathbf{e}_{\mathbf{i}} \mathbf{e}_{\mathbf{i}}{ }^{T}$, with tangent $\mathbf{e}_{3}$ representing a disk-shaped structure. The last term represents a ball tensor, $B=\lambda_{3} \sum_{i=1}^{3} \mathbf{e}_{\mathbf{i}} \mathbf{e}_{\mathbf{i}}{ }^{T}$, which is a structure with no preference of orientation. The eigenvalues in each tensorial term (Eq. 1) represent saliency measurements of surfaceness $\left(\lambda_{1}-\lambda_{2}\right)$, curveness $\left(\lambda_{2}-\lambda_{3}\right)$, and junctionness $\lambda_{3}$. Points with very small eigenvalues are regarded as noise.

Scalar information contained in an image needs to be encoded into a tensor before it can be used within the framework. A common approach is to define each voxel in the image as a token, and to assign a ball-shaped tensor, i.e no preferred initial orientation, to each of the tokens [14]. In our case, we make use of a priori information obtained from the analysis of the Hessian matrix to 1) reduce the number of tokens to be processed and 2) initialise each token with a first estimation of its orientation.

The analysis of the eigensystem of the Hessian matrix $H$ provides information about the orientations of structures within an image [8]. In the case of bright vessels with dark background, given $\left|\kappa_{3}\right| \geq\left|\kappa_{2}\right| \geq\left|\kappa_{1}\right|$ the eigenvalues of $H$, a voxel is said to belong to a vessel only if if $\kappa_{2}<0$ and $\kappa_{3}<0$ [9]. Its direction 
along the vessel is given by $\mathbf{v}_{\mathbf{1}}$ (the eigenvector associated with $\kappa_{1}$ ), when $\left|\kappa_{1}\right|$ is close to zero and $\left|\kappa_{3}\right| \approx\left|\kappa_{2}\right| \gg\left|\kappa_{1}\right|$.

Based on this information, voxels satisfying the condition $\kappa_{2}<0$ and $\kappa_{3}<0$ are considered for tensor voting, whereas the rest are rejected. The selected tokens are initialised by constructing a stick tensor $S$ at each location with orientation $\mathbf{v}_{\mathbf{1}}$. This is achieved by assigning (see Eq. 1):

$$
\left[\mathbf{e}_{\mathbf{1}}, \mathbf{e}_{\mathbf{2}}, \mathbf{e}_{\mathbf{3}}\right]=\left[\mathbf{v}_{\mathbf{1}}, \mathbf{v}_{\mathbf{2}}, \mathbf{v}_{\mathbf{3}}\right], \quad\left[\lambda_{1}, \lambda_{2}, \lambda_{3}\right]=\left[\left|\kappa_{1}\right|^{-1},\left|\kappa_{2}\right|^{-1},\left|\kappa_{3}\right|^{-1}\right]
$$

with $\mathbf{e}_{\mathbf{i}}, \lambda_{i}$ the eigen-vectors, -values from Eq. 1 and $\mathbf{v}_{\mathbf{i}}$ the eigenvectors of $H$.

Alternatively, it is possible to initialise the stick tensors with different weights reflecting an initial estimate of a token's vesselness. In this case, the response of a vesselness filter $\nu(\mathbf{x})$ is assigned to the first eigenvalue of the tensor $\mathbf{T}$, i.e. $\lambda_{1}=\nu(x)$. In our experiments, we obtained the best results by using the smoothed vesselness function proposed by Manniesing et al. [9] for initialisation.

Tensor Voting After $\mathbf{T}$ is decomposed into the three basic tensors, each token $\mathbf{p}$ propagates information to its neighbours in the form of a vote. A vote is a tensor that encodes the most likely direction of the normal at a neighbouring point. Votes are combined at every token to infer the type of structure going through it. More formally, the tensor voting at $\mathbf{p}$ is given by [14]:

$$
T V(\mathbf{p})=\sum_{\mathbf{q} \in \chi}\left(S V\left(\mathbf{v}, S_{\mathbf{q}}\right)+P V\left(\mathbf{v}, P_{\mathbf{q}}\right)+B V\left(\mathbf{v}, B_{\mathbf{q}}\right)\right)
$$

where $\chi$ denotes the neighbourhood of $\mathbf{p}, \mathbf{q}$ a point belonging to $\chi, S V, P V$ and $B V$ the stick, plate and ball votes cast to $\mathbf{p}$ by each component $S_{\mathbf{q}}, P_{\mathbf{q}}, B_{\mathbf{q}}$ of $\mathbf{q}$ and $\mathbf{v}=\mathbf{p}-\mathbf{q}$. The strength of the vote will be dependent on the norm of $\mathbf{v}$, as the influence of a point $\mathbf{q}$ should decay as its distance from $\mathbf{p}$ increases. The definition of points $\mathbf{q} \in \chi$ is performed by selecting a window of size $N$ within the image. Given $\sigma$, the scale used to obtain $H$, we define $N=2 \sigma$.

The tensor voting procedure can be regarded as a tensor convolution with a voting kernel. We refer the interested reader to $[13,14]$ for the mathematical derivation of $S V, P V$ and $B V$ in Eq. 2.

Voting Analysis As the result of the tensor voting is another tensor, it can be decomposed as in Eq. 1. Therefore, it can be decomposed as in Eq. 1 to construct three feature vector maps: the surface map (S-Map), the curve map (C-Map) and the junction map (J-Map). A voxel of these maps is a 2-tuple $(s, \mathbf{w})$, where $s$ is a scalar indicating strength and $\mathbf{w}$ is a unit vector indicating direction. Values for strength $s$ and direction $\mathbf{w}$ in the S-Map, C-Map and the J-Map are defined in the same way as saliency measurements and orientations for the $S, P$ and $B$ tensors, respectively.

In the context of our problem, we are interested in the information provided by the S-map (first term of Eq. 1). For a given tuple, we interpret $s$ as a consensus measurement of vesselness between a voxel and its neighbours and $\mathbf{w}$ as the direction along the vessel. 
Data Fusion Given two $D$ dimensional vectors, the cosine of the angle between them is an index on the extent to which they are aligned. As vessels are welloriented structures, the cosine of the direction vectors is a surrogate of vesselness consistency between different images. Given two sets of tuples $\left(s_{1}, \mathbf{w}_{\mathbf{1}}\right),\left(s_{2}, \mathbf{w}_{\mathbf{2}}\right)$ from vesselness maps obtained from two different modalities after voting analysis, with $\left\|\mathbf{w}_{\mathbf{1}}\right\|=\left\|\mathbf{w}_{\mathbf{2}}\right\|=1$, it is possible to fuse the maps into a single one through the following expression:

$$
\varphi(\mathbf{p})=0.5\left|\mathbf{w}_{\mathbf{1}} \cdot \mathbf{w}_{\mathbf{2}}\right|\left(s_{1}+s_{2}\right)
$$

The fusion scheme is a measure rewarding consensus: it becomes an average when there is total direction agreement and punishes discord by reducing the absolute value of the output.

Multi-scale Framework By redefining Eq. 3 as a function of the scale $\sigma$ used for token initialisation through analysis of $H$ :

$$
\varphi(\mathbf{p}, \sigma)= \begin{cases}0.5 \cdot\left|\mathbf{w}_{\mathbf{1}} \cdot \mathbf{w}_{\mathbf{2}}\right| \cdot\left(s_{1}+s_{2}\right) & \text { if } \kappa_{2}<0 \cap \kappa_{3}<0 \\ 0 & \text { otherwise }\end{cases}
$$

it is possible to reformulate the tensor voting scheme into a multi-scale framework. The reformulated measurement from Eq. 4 is obtained at different scales $\sigma$. A final estimate is obtained by retaining the maximum response at different scales:

$$
\varphi(\mathbf{p})=\max _{\sigma_{\min } \leq \sigma \leq \sigma_{\max }} \varphi(\mathbf{p}, \sigma)
$$

where $\sigma_{\min }-\sigma_{\max }$ is the range of scales in which it is expected to find vessels.

SEEG Planning System Integration The resulting vessel probability map, $\varphi(\mathbf{p})$, is used as an input of our computer assisted planning system [12]. As the electrode implanting trajectory needs to be further than a safety margin from the critical tissue (vessels in this case), the probability map serves as a measure of risk of crossing a vessel. Within the planning system, the probability map is converted into a 3D surface mesh object, coloured using a pre-defined landmark colour scheme [12] and displayed within the neuronavigation planning system along with other critical brain structures (Figure 2(i)).

\section{$3 \quad$ Validation and Results}

Data. Twelve paired datasets from two image modalities available within the planning system, 3D phase contrast MRI (3DPC) and CT Angiograms (CTA), were used in this work (informed consent obtained from all the patients). The $3 \mathrm{DPC}$ data were acquired on a $1.5 \mathrm{~T}$ Siemens Avanto MR scanner with voxel size resolution $0.8593 \times 0.8593 \times 1 \mathrm{~mm}^{3}$ and velocity encoding of $5 \mathrm{~cm} / \mathrm{s}$ in each direction. CTA images were acquired on a Siemens SOMATOM Definition AS+ scanner with voxel size resolution $0.4296 \times 0.4296 \times 0.75 \mathrm{~mm}^{3}$. 
Gold Standard Generation. Three different observers (a neurosurgical trainee, a physicist with 8-year experience in clinical neuroimaging and a master student trained for the task) annotated blood vessels structures following the protocol typically used in clinical practice for SEEG planning in the absence of a computer assisted planning system. The annotation procedure was as follows:

1. An intracranial space mask was applied to the CTA image to remove skull and to the 3DPC image to remove extracranial blood vessels.

2. Masked CTA and 3DPC were separately thresholded to give an initial estimate of the vessels. The threshold was defined by visually evaluating the resulting segmentation and determining if noise and blood vessels were easy to distinguish and differentiate from each other with minimal manual cleaning.

3. Small isolated clusters were removed by diameter size within MeshLab. The observers varied the threshold until they considered the segmented result satisfactory through visual inspection. Afterwards, large noise (e.g. calcifications) was removed by manually editing the images using MeshLab.

The six segmentations of the observers were combined into a consensus agreement through a voting strategy.

Validation Scheme. The proposed algorithm was evaluated on the twelve affinely co-registered datasets using ten different scales exponentially distributed between $\sigma_{\min }=1.0$ and $\sigma_{\max }=4.5$. The size of the neighbourhood window $N$ was varied accordingly $(N=2 \sigma)$. In order to compute the vesselness function $\nu(\mathbf{x})$ required for tensor initialisation, we followed the guidelines reported in [9].

For a quantitative evaluation, the binarised vessel image $S$ was compared to the consensus segmentation $M$ using the Dice similarity coefficient $D S C=$ $2|S \cap M| /|S+M|$. We used the DSC to assess the performance of our method and that one of each observer w.r.t the consensus when doing a semi-automated segmentation with a single modality, as it is done in clinical routine, thus comparing the accuracy of the proposed method to current practice.

Results and Discussion. The mean Dice coefficients obtained from comparing our method and the observer's annotations to the consensus segmentation are summarised in Table 1. The DSC of the proposed multi-modal approach is superior to that one obtained by the best performing rater using a single modality. Although CTA images have richer vessel content that is reflected in better rater segmentations, 3DPC contains complementary information that is exploited by the proposed algorithm. An example of this behaviour is illustrated in Figure 2 where small vessels (absent in 3DPC) and the superior sagittal sinus (with a weak signal in CTA) are both appearing in the final result.

A visual comparison of obtained vesselness maps with a consensus map is given in Figure 2(e-h) to further illustrate the performance of our method w.r.t. the current semi-automated approach. Although the proposed algorithm is more prone to false positives, it also achieves a better detection of vessel branches 
Table 1. Mean \pm standard deviation of the Dice similarity coefficient (DSC) when comparing our method and the observers annotations to a consensus segmentation.

\begin{tabular}{|c|c|c|c|c|c|c|c|}
\hline \multirow{2}{*}{$\begin{array}{c}\text { Our } \\
\text { method }\end{array}$} & \multicolumn{2}{|c|}{ Observer 1 } & \multicolumn{2}{c|}{ Observer 2 } & \multicolumn{2}{c|}{ Observer 3 } \\
\cline { 3 - 8 } & 3DPC & CTA & 3DPC & CTA & 3DPC & CTA \\
\hline DSC & $0.88 \pm 0.02$ & $0.32 \pm 0.16$ & $0.74 \pm 0.03$ & $0.35 \pm 0.10$ & $0.78 \pm 0.02$ & $0.35 \pm 0.09$ & $0.77 \pm 0.02$ \\
\hline
\end{tabular}

than the semi-automated approach. Under the scope of trajectory planning, it is preferable to have a high sensitivity, at the cost of false positives, than missing any critical structure.

\section{Conclusions}

In this paper, we have presented a vessel extraction method for the identification of critical landmarks within a computer assisted SEEG planning system. The main feature of this method is that it integrates scale, neighbouring structure and feature stability within a single framework. The introduction of a voting neighbourhood within the well-established multi-scale approach, and the use of complimentary sources of information reduces noisy structures and improves the connectivity of the voxels belonging to vessels. The results presented here demonstrate the superiority of our method to the semi-automated single-modality segmentation, indicating the possibility of safer SEEG planning, with reduced patient morbidity.

Acknowledgements. This work is supported by the Department of Health and Wellcome Trust through the Health Innovation Challenge Fund (HICF-T4-275), the EPSRC (EP/H046410/1, EP/J020990/1, EP/K005278), the MRC (MR/J01107X/1),

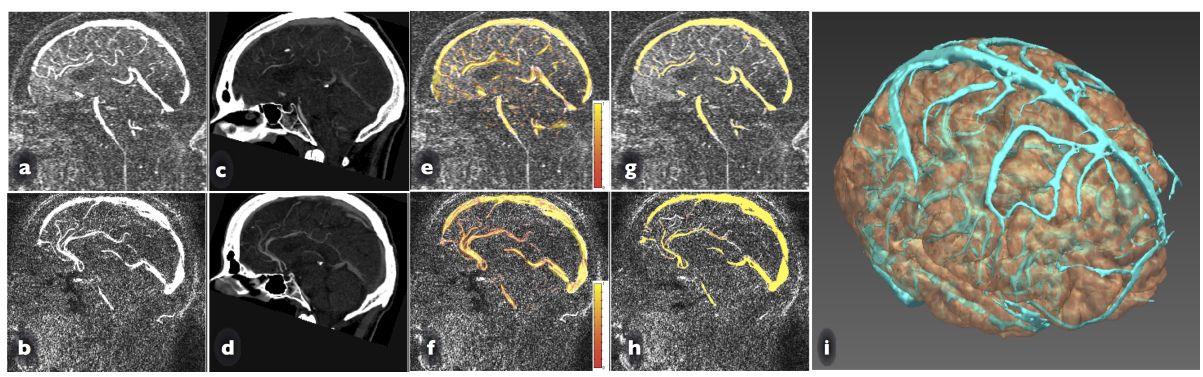

Fig. 2. (a-b) 3DPC and (c-d) CTA images, superposed vesselness map generated by the proposed method over (e-f) 3DPC and (g-h) consensus segmentation for two subjects. i) Integrated visualisation in the SEEG planning system. 
the EU-FP7 project VPH-DARE@ IT (FP7-ICT-2011-9-601055), the National Institute for Health Research Biomedical Research Unit (NIHR BRU) in Dementia at University College London Hospitals NHS Foundation Trust and University College London, and the NIHR University College London Hospitals Biomedical Research Centre (BRC) (UCLH/UCL High Impact Initiative). The authors thank Dr Mark White from NHNN for the provided support.

\section{References}

1. David, O., Blauwblomme, T., Job, AS., Chabardès S., Hoffmann, D., Minotti, L., Kahane, P.: Imaging the seizure onset zone with stereo-electroencephalography. Brain 134, 2898-2911 (2011)

2. Olivier A., Boling WW., Tanriverdi T.: Techniques in epilepsy surgery: the MNI approach. Cambridge University Press, Cambridge (2012)

3. Bériault, S., Al Subaie, F., Collins, DL., Sadikot, AF., Pike, GB.: A multi-modal approach to computer-assisted deep brain stimulation trajectory planning. Int J CARS 7, 687-704 (2012)

4. Essert C., Haegelen C., Lalys F., Abadie A., Jannin P.: Automatic computation of electrode trajectories for Deep Brain Stimulation: a hybrid symbolic and numerical approach. Int J Comput Assist Radiol Surg 7(4), 517-532 (2012)

5. Shamir RR., Joskowicz L., Tamir I., Dabool E., Pertman L., Ben-Ami A., Shoshan Y.: Reduced risk trajectory planning in image guided keyhole neurosurgery. Med Phys 39, 2885-2895 (2012)

6. Du, X., Ding, H., Zhou, W., Zhang, G., Wang, G.: Cerebrovascular segmentation and planning of depth electrode insertion for epilepsy surgery. Int J CARS 8, 905916 (2013)

7. Lesage, D., Angelini, ED., Bloch, I., Funka-Lea, G.: A Review of 3D Vessel Lumen Segmentation Techniques: Models, Features and Extraction Schemes. Med Image Anal 13, 819-845 (2009)

8. Frangi, AF., Niessen, WJ., Vincken KL., Viergever, MA.: Multiscale Vessel Enhancement Filtering. In: Wells, WM., Colchester, A., Delp, SL. (eds) MICCAI 1998, LNCS 1496, Springer Verlag, pp. 130-137 (1998)

9. Manniesing, R., Viergever, MA., Niessen, WJ.: Vessel enhancing diffusion: A scale space representation of vessel structures. Med Image Anal 10, 815-825 (2006)

10. Passat, N., Ronse, C., Baruthio, J., Armspach, J-P., Foucher, J.: Watershed and multimodal data for brain vessel segmentation: Application to the superior sagittal sinus. Image Vision Comput 25, 512-521 (2007)

11. Hu, Z., Niemeijer, M., Abràmoff, MD., Garvin, MK.: Multimodal Retinal Vessel Segmentation From Spectral-Domain Optical Coherence Tomography and Fundus Photography. IEEE Trans Med Imag 31, 1900-1911 (2012)

12. Zombori, G., Rodionov, R., Nowell, M., Zuluaga, MA., Clarkson, M., Micallef, C., Diehl, B., Wehner, T., Miserocchi, A., McEvoy, A., Duncan, J., Ourselin, S.: A computer assisted planning system for the placement of sEEG electrodes in the treatment of epilepsy. In: IPCAI 2014, (In press)

13. Medioni, G., Lee, M-S., Tang, C-K.: A Computational Framework for Segmentation and Grouping. Elsevier Science (2000)

14. Moreno, R., Garcia, MA., Puig, D., Pizarro, L., Burgeth, B., Weickert, J.: On improving the efficiency of tensor voting. IEEE Trans Pattern Anal Machine Intell $33,2215-2228$ (2011) 